\title{
Effect of the Base-band Measurement Setup Errors on DPD Performance and Elimination Procedure Ahmet Hayrettin YUZER ${ }^{1}$ and Emine Betul SANLI ${ }^{2}$ \\ ${ }^{1}$ Department of Electrical and Electronics Engineering, University of Karabuk, Karabuk, Turkey \\ ${ }^{2}$ Department of Mechatronics Engineering, Karabuk University ,Karabuk, Turkey \\ *corresponding author,E-mail: hayrettinyuzer@karabuk.edu.tr
}

\begin{abstract}
In this study, the effect of base-band measurement setup errors on DPD performance was investigated and a calibration procedure is developed to eliminate the measurement errors. A base-band measurement setup is prepared at laboratory with instruments and then the data which is measured and the deteriorating effect of errors on Digital Predistortion (DPD) linearization performance are investigated. In order to eliminate deteriorating effect of this error a three steps calibration procedure is developed. Before and after calibration application DPD performance is measured. It is showed both in simulation and experimentally that the calibration procedure improved the DPD system linearization performance from $10 \mathrm{~dB}$ to $26 \mathrm{~dB}$ and $13 \mathrm{~dB}$ to $20 \mathrm{~dB}$, respectively.
\end{abstract}

\section{Introduction}

RF power amplifiers are (PA) widely used in telecommunications systems. In order to get maximum power, PA is derived near saturated power. Inherently generated intermodulation (IMD) signals in all PA which is derived near $\mathrm{P} 1 \mathrm{~dB}$ deteriorate the adjacent channel interference $(\mathrm{ACI})$ and transmitter efficiency for the PA using in wireless system [1]. Modern communication systems have special standards like IEEE802.11ac. According to those standards IMD level should below then the predefined value in the communication mask [2]. Hence, RF power amplifier performance has important effect on overall communication system performance. In order to decrease IMD level to be in the communication mask there is a number of linearization technique reported in literature, like digital pre-distortion (DPD). Because first step of DPD is the extract acceptable amplifier model, these days, there is growing demand on power amplifier (PA) modelling in order to get best DPD linearization performance and increase the efficiency of amplifier [3-6]. A measurement setup to measure amplifier response is needed for modelling.

It is reported in literature that the nonlinearity due to software program and hardware system of the instrument are deteriorate the measurement results [2-8].

In this study, a base-band measurement setup is prepared in order to investigate the effects of measurement setup errors on the modelling and the DPD performance and then a calibration procedure is developed. This measurement setup is explained in the next section. Then measurement errors are explained in the third section. Calibration procedure, theory of behavioural modelling and DPD are explained in the fourth and fifth section. After calibration process, the reported measurement errors are diminished and improvement in the DPD performance is showed in the fifth section.

\section{Base-band I/Q measurement setup}

Base-band measurement setup was used to measure corresponding amplifier response for the excitation in order to extract amplifier model. Input and output measured data are base-band I/Q data. Base-band input and output I/Q data are enough to get behavioral model of the amplifier since DPD system is working on digital data. In other words, there is no need to measure carrier signal. Benchmark for the DPD system performance is described according to improvement ratio in the ACPR value numerically and decreasing IMD level on the spectrum analyzer screen visually. A general base-band measurement setup or a specially prepared setup can be used to measure amplifier response. General base-band measurement setup [9] which is given at Fig. 1 is composed of a Vector Signal Generator (VSG), a Vector Signal Analyzer (VSA) and a computer to download IQ data to VSG and retrieve amplifier response as IQ data from VSA to the computer. While some of researchers are using this setup with "Agilent 89600 VSA SOFTWARE" $[10,11]$, some of researchers are preparing their own measurement setup [12-16].

Only uncalibrated base-band IQ data can be taken from Vector Signal Analyzer-VSA (Agilent- EXA N9010A) instrument without using expensive VSA Software. These data deteriorate the DPD performance since the data are not calibrated.

A calibration procedure is developed in the MATLAB program to take base-band data from VSA and then calibrate it without using Agilent VSA Software. Firstly a measurement system is prepared at laboratory and the photograph of the measurement system is given at Fig. 2. In the measurement setup a Agilent- 8267D as Vector Signal Generator-VSG and a Agilent- EXA N9010A as Vector Signal Analyzer-VSA instruments were used in order to generate and measure base-band data at this setup. In addition to these instruments, a computer was used to 
generate base-band I/Q waveform and to download it to the VSG then to retrieve base-band I/Q data from the VSA.

Data downloading and retrieving operation was performed by a MATLAB based program instead of the "Agilent 89600 VSA SOFTWARE". An amplifier having $47 \mathrm{dBm}$ output saturation power and working at $1 \mathrm{MHz}$ to $40 \mathrm{MHz}$ frequency range was used. $10 \mathrm{MHz}$ reference cable and Trigger cable connected to the instruments for time alignment. Two different connection, state-1 and state-2, were used in order to calibrate the system and measure the amplifier response.

Digitally modulated QAM64 signal having 800.000 sample is used as base-band excitation data. Hereafter this signal is called as input/excitation signal.

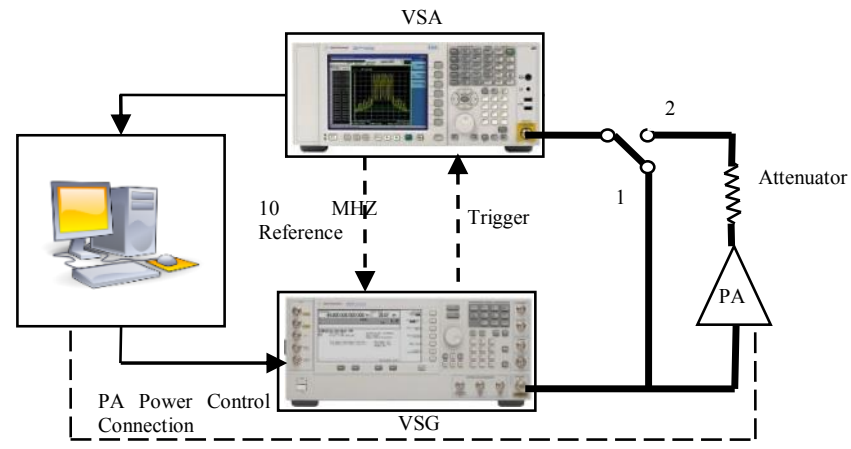

Fig. 1 General Base-band Measurement Setup [9]

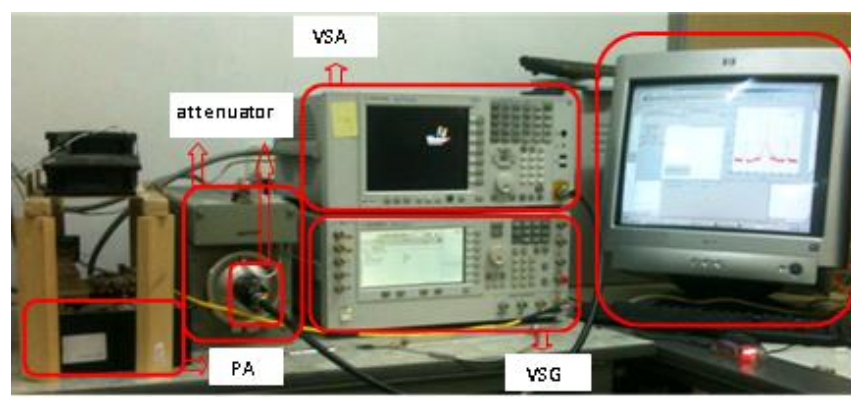

Fig. 2 Measurement Setup

\section{Measurement Errors}

Firstly, the measurement connection cable has been taken at state-1 which means that the input signal generated from the VSG was directly measured from the VSA. Although there are a $10 \mathrm{MHz}$ reference and trigger, there can be a misalignment between the downloaded and the measured signals. This is why a time alignment was performed. The magnitude of the digitally generated (downloaded to the VSG), measured data and the zoomed version were plotted as seen in Fig. 3.

One can easily observe that there is a difference between the loaded and the measured data. This means that the amplifier is excited with a signal different than the one that was loaded. Hence, it is almost impossible to find a better model by using loaded data to increase ACPR by using DPD system. The data plotted on Fig. 3 are normalized to its maximum value. The maximum error in the magnitude is $\pm 0.1 \mathrm{~V}$ and the maximum phase error is $\pm 20^{\circ}$ as it can be seen from Fig. 4 and Fig. 5. This much error is unacceptable, especially by considering the ACPR between fundamental signal and intermodulation (IMD) signal is approximately $30 \mathrm{dBc}$. As a result, this situation result in indispensable performance deterioration in DPD. Therefore, calibration procedure is foregone conclusion.

Since it is out of scope of this study, the source of errors was not investigated. In addition, it is thought that this error is not resulted from I/Q imbalance as investigated in [17]. It is presumed that distortion is generated by the amplifier at the output stage of the instrument in high power output situation.

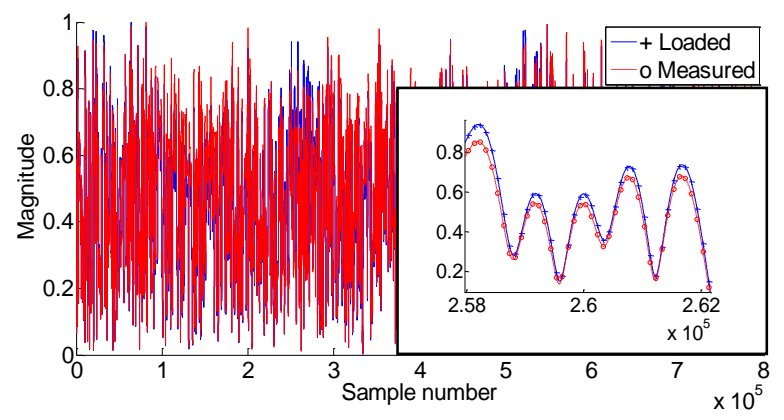

Fig. 3 Magnitude of loaded and time aligned measured data under connection-1

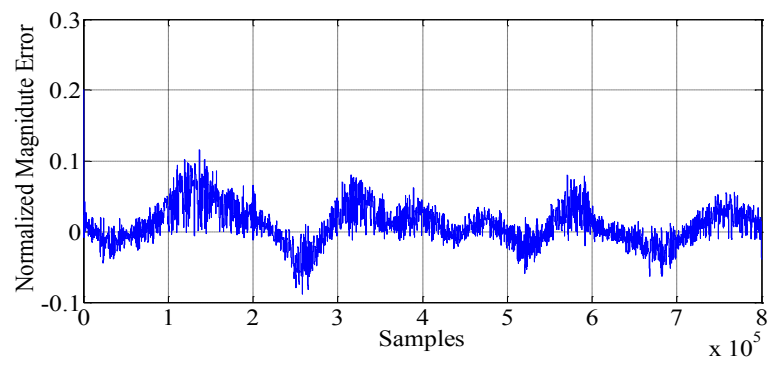

Fig. 4 Magnitude error between loaded and time aligned measured data under connection-1

\section{Calibration}

A calibration procedure was developed, in order to get rid of the measurement errors, both in magnitude and in phase. The calibration procedure consists of 3 steps: magnitude calibration, phase calibration and testing. The measurement setup connection is taken to the state- 1 and then three measurements at three different power levels are performed.

i. The magnitudes of the loaded data and the first measurement results are compared. The errors between these signals are stored as "magnitude correction ratio array".

ii. The phases of the loaded data and the second measurement results are compared. The errors between these signals are stored as "phase correction value array".

iii. The magnitude of the third measurement results are multiplied, sample by sample, with the "magnitude 
correction ratio array". Then the phase of the third measurement result is shifted as "phase correction value array", sample by sample. The magnitude and the phase errors between the loaded data and the third measurement data on which magnitude and phase calibration was applied are compared.

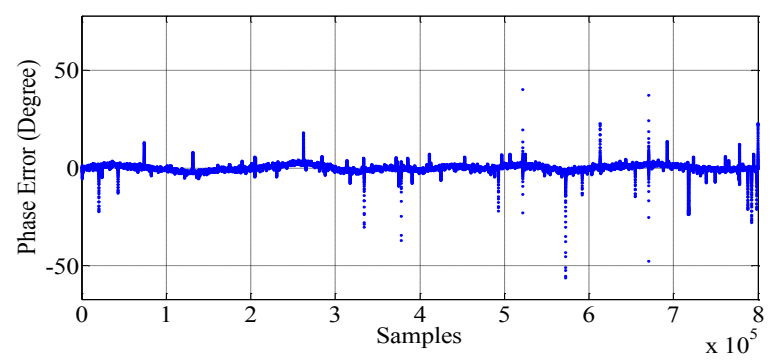

Fig. 5 Phase error between loaded and time aligned measured data under connection-1

After calibration, magnitudes of loaded and measured signal, magnitude error and phase error are plotted as it can be shown from, Fig. 6, Fig. 7 and, Fig. 8 respectively. It is seen from these graph that majority of errors are eliminated. After calibration, the maximum error in the magnitude is decreased from $\pm 0.1 \mathrm{~V}$ to $\pm 0.002 \mathrm{~V}$ and the maximum phase error is decreased from $\pm 20^{\circ}$ to \pm $3^{\circ}$.

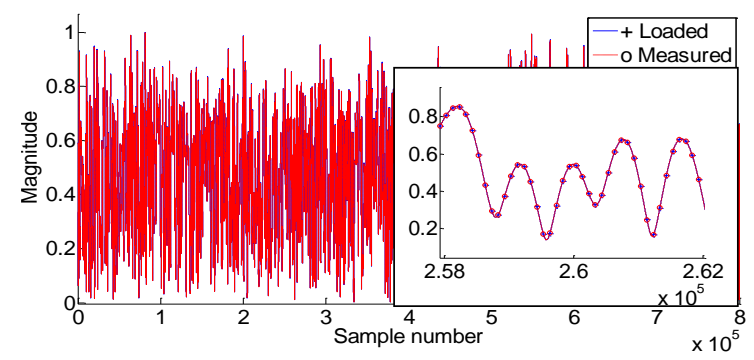

Fig. 6 Magnitude of loaded and time aligned measured data after calibration

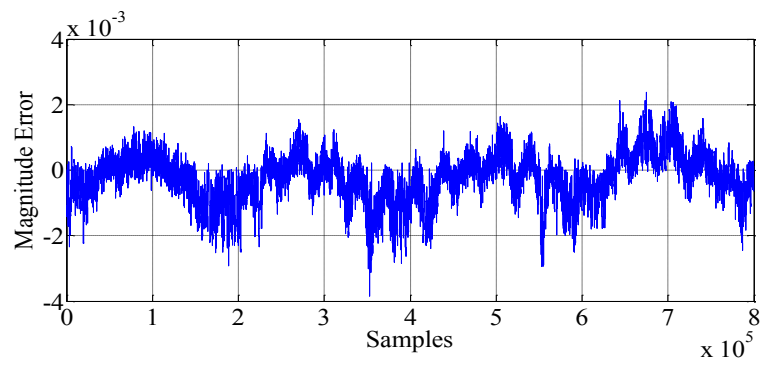

Fig. 7 Magnitude error after calibration

\section{Behavioral Modelling and DPD}

\subsection{Modelling with memory polynomial}

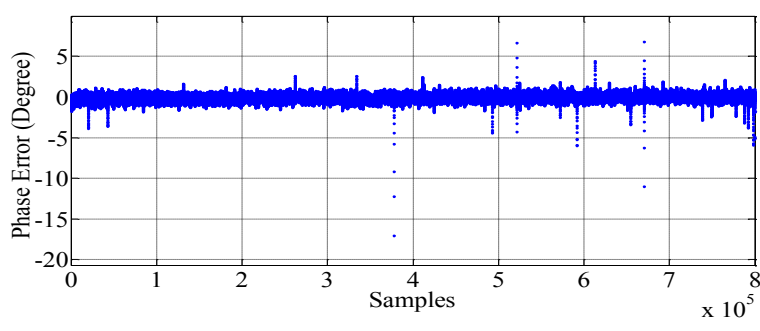

Fig. 8 Phase error after calibration

There are a number of polynomial modelling techniques in the scientific literature. In this study, the Unit Delay Memory Polynomial modelling (MPM-[18]) technique was used to get the model of PA. Mathematical representation of model is given in (1).

$$
\mathrm{z}[\mathrm{n}]=\sum_{\mathrm{q}=0}^{\mathrm{M}-1} \sum_{k=1}^{K} a_{k, q}|x[n-q]|^{2(k-1)} x[n-q]
$$

where $x[n]$ represents the $I / Q$ input signal, $z[n]$ represents the $\mathrm{I} / \mathrm{Q}$ output signal, $\mathrm{a}_{\mathrm{k}, \mathrm{q}}$ represents the modelling polynomial coefficients $(\mathrm{M} \times \mathrm{K}), \mathrm{K}$ represents the polynomial order and $\mathrm{M}$ represents the number of delay line.

\subsection{Digital predistortion}

DPD is one of the preferred linearization techniques because of surpassing properties such as general system efficiency, application performance and adaptability. The structure of a linearization was frequently explained in literature $[10,18$ 23]. Briefly, a DPD system is modeled as a black box, namely a Pre-distorter (PD) having nonlinear characteristic, which is cascade connected to the PA. The main function of a PD block is to distort the input signal before exciting the amplifier such that the overall system response will become linear. DPD system block diagram is given in Fig. 9. PD input-output characteristics is just inverse of PA inputoutput characteristics as shown in Fig. 10. Although, only PD block (modelling function and coefficients) is enough to apply real DPD system, PA modeling is necessary for DPD system simulation. In this study, a special MATLAB program is prepared to simulate DPD system by using loaded data and the measured PA response data.

While simulating the DPD system, measured data are needed. To do so, firstly calibration process is performed, then connection is taken at state- 2 and amplifier response to the excitation signal is measured as I/Q data. Amplifier model is extracted (model coefficients which are defined in (1) are found) by using input data as $x[n]$ and output data as $\mathrm{z}[\mathrm{n}]$ in (1). This model is named as Forward model and only be used in DPD simulation. As a next step, namely Inverse model is extracted by taking input data as $z[n]$ and output data as $x[n]$ in (1). Inverse model (PD block) is used both for simulation and real DPD system. The relation between $x[n]$ and $z[n]$ is nonlinear. There is similar relation between $V_{i-p d}$ and $\mathrm{V}_{\mathrm{o}-\mathrm{pd}}$ as shown in Fig. 10. Similarly, the relation between $\mathrm{v}[\mathrm{n}]$ and $\mathrm{x}[\mathrm{n}]$ is nonlinear and similar relation 
between $\mathrm{V}_{\mathrm{i}}$ and $\mathrm{V}_{\mathrm{i} \text {-pd }}$ as shown in Fig. 10. Finally, overall expected DPD system relation between $v[n]$ (input of the DPD system) and $z[n]$ (output of the DPD system) will become linear.

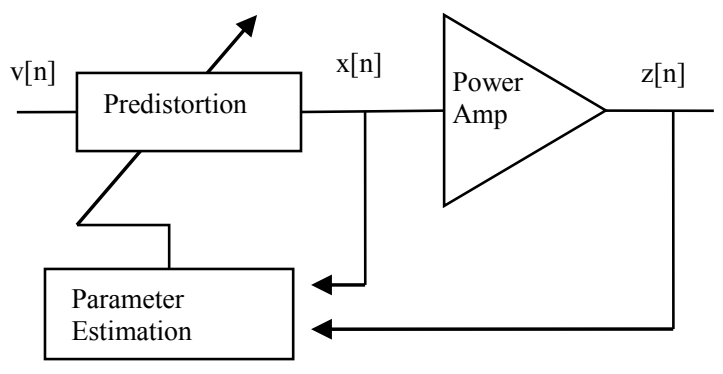

Fig. 9 Generic predistortion scheme [22]

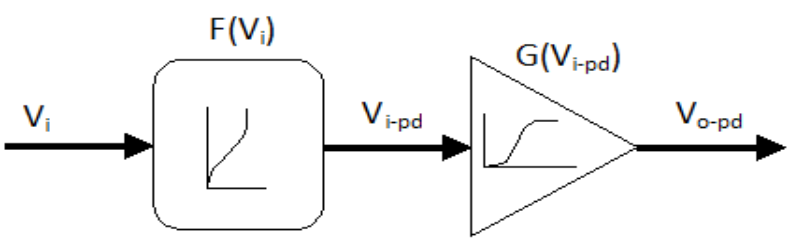

Fig. 10 Overall DPD system (Mekechuk et al., 2004).

\section{Discussion}

The modeling accuracy can be evaluated by using the normalized mean square error (NMSE) parameter which is defined at [24].

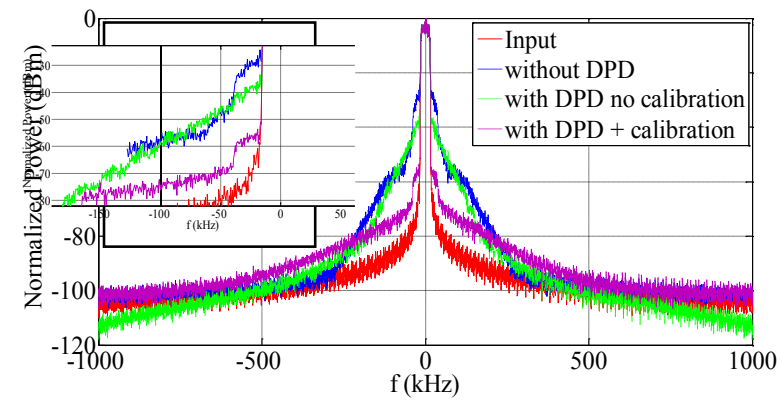

Fig. 11 Frequency spectrum of the measured data (input and output) and simulated data (un-calibrated and calibrated DPD output)

This evaluation is applied both for calibrated and uncalibrated data. Frequency spectrum of the measured input and output, simulated DPD output for the un-calibrated system and DPD output for the calibrated system is given in Fig. 11. NMSE values of the without calibration situation and the with-calibration situation are $-26.57 \mathrm{~dB}$ and -33.97 $\mathrm{dB}$, respectively.

In a real measurement setup, the input is applied to the Inverse model [25]. Output of the inverse model, distorted signal, is loaded to the VSG then it is applied to the PA. Afterwards, the PA response is measured both for calibrated and un-calibrated distorted input data. The VSA screen is saved while the VSA is working for the spectrum sweeping mode, to show the spectrum of input, output, DPD output for the un-calibrated system and the DPD output for the calibrated system as given in Fig. 12. ACPR improvement ratio is tabulated as given in Table 1.

Table 1 DPD Performance comparison with PA

\begin{tabular}{|c|c|c|}
\cline { 2 - 3 } \multicolumn{1}{c|}{} & $\begin{array}{c}\text { Without } \\
\text { Calibration }\end{array}$ & $\begin{array}{c}\text { With } \\
\text { Calibration }\end{array}$ \\
\hline $\begin{array}{c}\text { Simulated DPD } \\
\text { improvement }\end{array}$ & $9.75 \mathrm{~dB}$ & $26 \mathrm{~dB}$ \\
\hline $\begin{array}{c}\text { Real System } \\
\text { DPD } \\
\text { improvement }\end{array}$ & $12.99 \mathrm{~dB}$ & 20.334 \\
\hline
\end{tabular}

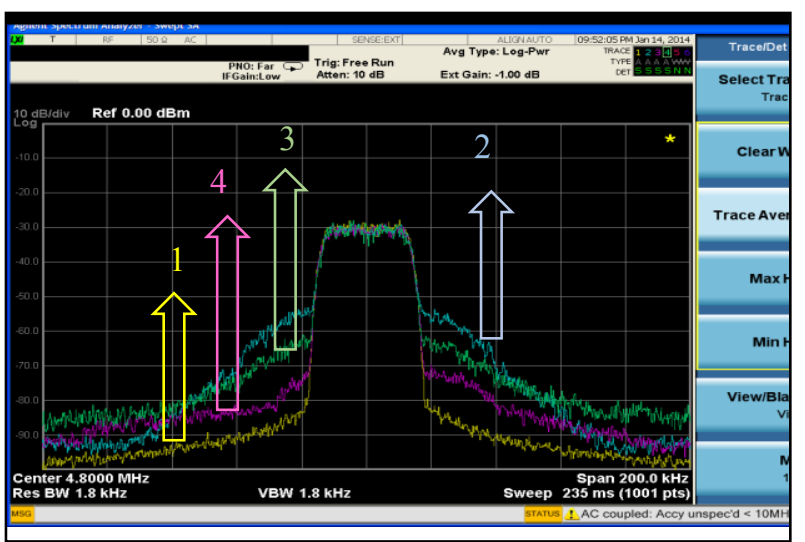

Fig. 12 VSA screen showing frequency spectrum of input(yellow-1), output(blue-2) and un-calibrated DPD output(green-3) and calibrated DPD output(magenta-4).

\section{Conclusions}

In this study, the effect of base-band measurement setup errors on DPD performance was investigated and a calibration process is developed. General base-band measurement setup of which details are explained in literature is used to measure base-band signal. Measurement setup connection is taken at state-1, the signal generated by VSG is measured. It is realized that there is difference between loaded signal and measured signal. Although the source of errors was not investigated, since it is out of scope of this study, it is presumed that the distortion is generated by the amplifier at the output stage of the instrument in high power output situation. These distortions are being eliminated with the calibration process developed under this study. This calibration process is the alternative way of expensive options for error elimination for this type of measurement. 


\section{Acknowledgements}

This work is supported by Coordinatorship of Karabük University's Scientific Research Projects(KBÜ-BAP-13/2YL-046) Karabük, TURKEY

\section{References}

[1] J. S. Kenney and P. Fedorenko, "Identification of RF Power Amplifier Memory Effect Origins using ThirdOrder Intermodulation Distortion Amplitude and Phase Asymmetry," in Microwave Symposium Digest, 2006. IEEE MTT-S International, 2006, pp. 1121-1124.

[2] K. Freiberger, M. Wolkerstorfer, H. Enzinger, and C. Vogel, "Digital predistorter identification based on constrained multi-objective optimization of WLAN standard performance metrics," in 2015 IEEE International Symposium on Circuits and Systems (ISCAS), 2015, pp. 862-865.

[3] J. H. K. Vuolevi, T. Rahkonen, and J. P. A. Manninen, "Measurement technique for characterizing memory effects in RF power amplifiers," IEEE Trans. Microw. Theory Tech., vol. 49, no. 8, pp. 1383-1389, Aug. 2001 .

[4] S. Boumaiza, M. Helaoui, O. Hammi, T. Liu, and F. M. Ghannouchi, "Systematic and Adaptive Characterization Approach for Behavior Modeling and Correction of Dynamic Nonlinear Transmitters," IEEE Trans. Instrum. Meas., vol. 56, no. 6, pp. 2203-2211, Dec. 2007.

[5] H. Ku and J. S. Kenney, "Behavioral modeling of nonlinear RF power amplifiers considering memory effects," IEEE Trans. Microw. Theory Tech., vol. 51, no. 12, pp. 2495-2504, Dec. 2003.

[6] P. Draxler, J. Deng, D. Kimball, I. Langmore, and P. M. Asbeck, "Memory effect evaluation and predistortion of power amplifiers," in Microwave Symposium Digest, 2005 IEEE MTT-S International, 2005, pp. 1549-1552.

[7] W. Bosch and G. Gatti, "Measurement and simulation of memory effects in predistortion linearizers," IEEE Trans. Microw. Theory Tech., vol. 37, no. 12, pp. 1885-1890, Dec. 1989.

[8] G. Kompa and F. van Raay, "Error-corrected largesignal waveform measurement system combining network analyzer and sampling oscilloscope capabilities," IEEE Trans. Microw. Theory Tech., vol. 38, no. 4, pp. 358-365, Apr. 1990.

[9] C. Luque, "Model-based pre-distortion for signal generators," Dept. of Technology, University of Gavle, Sweden, 2007.

[10] Y.-J. Liu, J. Zhou, W. Chen, and B.-H. Zhou, "A Robust Augmented Complexity-Reduced Generalized Memory Polynomial for Wideband RF Power Amplifiers," IEEE Trans. Ind. Electron., vol. 61, no. 5, pp. 2389-2401, May 2014.

[11] M. V. Amiri, M. Helaoui, and F. M. Ghannouchi, "Streamlined MIMO cross-over digital predistortion," in 2014 IEEE Radio and Wireless Symposium (RWS), 2014, pp. 283-285.
[12] S. Amin, P. N. Landin, P. Handel, and D. Rönnow, "Behavioral Modeling and Linearization of Crosstalk and Memory Effects in RF MIMO Transmitters," IEEE Trans. Microw. Theory Tech., vol. 62, no. 4, pp. 810823, Apr. 2014.

[13] M. V. D. Nair, R. Giofrè, L. Piazzon, and P. Colantonio, "A comparative study on digital predistortion techniques for Doherty amplifier for LTE applications," in 2014 International Workshop on Integrated Nonlinear Microwave and Millimetre-wave Circuits (INMMiC), 2014, pp. 1-3.

[14] N. Naraharisetti, P. Roblin, C. Quindroit, M. Rawat, and S. Gheitanchi, "2D quasi exact inverse of PA model in digital predistorter for concurrent dual-band system," in Wireless and Microwave Technology Conference (WAMICON), 2014 IEEE 15th Annual, 2014, pp. 1-4.

[15] H. Watanabe, T. Fukami, H. Saito, A. Tomiki, O. Ceylan, H. Nunomura, O. Shigeta, T. Shinke, and K. Kojima, "High speed downlink system for small satellite and high-efficiency x-band GaN SSPA," in Microwave Symposium (IMS), 2014 IEEE MTT-S International, 2014, pp. 1-4.

[16] H. Sarbishaei, B. Fehri, Y. Hu, and S. Boumaiza, "Dual-Band Volterra Series Digital Pre-Distortion for Envelope Tracking Power Amplifiers," IEEE Microw. Wirel. Compon. Lett., vol. 24, no. 6, pp. 430-432, Jun. 2014.

[17] M. SHAH and S. GUPTA. "Baseband I/Q regeneration Method for Direct Conversion Receiver to nullify effect of I/Q mismatch". Advanced Electromagnetics, [S.1.], v. 5, n. 3, p. 50-55, nov. 2016. ISSN 2119-0275. Available

$<$ http://www.aemjournal.org/index.php/AEM/article/vi ew/435>. Date accessed: 27 july 2017. doi: http://dx.doi.org/10.7716/aem.v5i3.435.

[18] Cruz Nuñez-Perez, J., Ricardo Cardenas-Valdez, J., Gontrand, C., Apolinar Reynoso-Hernandez, J., Iwao Hirata-Flores, F., Jauregui-Duran, R. and J. PerezPinal, F. (2013) 'Flexible test bed for the behavioural modelling of power amplifiers', COMPEL - The international journal for computation and mathematics in electrical and electronic engineering, 33(1/2), pp. 355-375. doi: 10.1108/compel-11-2012-0326.

[19] G. C. L. Cunha, S. Farsi, B. Nauwelaers, and D. Schreurs, "An FPGA-based digital predistorter for RF power amplifier linearization using cross-memory polynomial model," in 2014 International Workshop on Integrated Nonlinear Microwave and Millimetrewave Circuits (INMMiC), 2014, pp. 1-3.

[20] W. Chen, S. Zhang, Y.-J. Liu, F. M. Ghannouchi, Z. Feng, and Y. Liu, "Efficient Pruning Technique of Memory Polynomial Models Suitable for PA Behavioral Modeling and Digital Predistortion," IEEE Trans. Microw. Theory Tech., vol. 62, no. 10, pp. 2290-2299, Oct. 2014.

[21] R. G. Bozomitu, V. Cehan, and V. Popa, "A New Linearization Technique Using Multi-sinh Doublet," 
Adv. Electr. Comput. Eng., vol. 9, no. 2, pp. 45-57, 2009.

[22] D. R. Morgan, Z. Ma, J. Kim, M. G. Zierdt, and J. Pastalan, "A Generalized Memory Polynomial Model for Digital Predistortion of RF Power Amplifiers," IEEE Trans. Signal Process., vol. 54, no. 10, pp. 38523860, Oct. 2006.

[23] K. Mekechuk, W.-J. Kim, S. P. Stapleton, and J. H. Kim, "Linearizing power amplifiers using digital predistortion, eda tools and test hardware," High Freq. Electron., vol. 3, no. 4, pp. 18-25, 2004.

[24] P. Suryasarman and A. Springer, "Adaptive digital predistortion for multiple antenna transmitters," in 2013 IEEE Global Conference on Signal and Information Processing (GlobalSIP), 2013, pp. 1146-1149.

[25] I. J. Bahl, "Linearization techniques," Fundam. RF Microw. Transistor Amplif., pp. 331-361, 2008. 